\title{
Newborn Care Practices among Mother-Infant Dyads in Urban Uganda
}

\author{
Violet Okaba Kayom, ${ }^{1}$ Abel Kakuru, ${ }^{2}$ and Sarah Kiguli ${ }^{1}$ \\ ${ }^{1}$ Department of Paediatrics and Child Health, Makerere University College of Health Sciences, Kampala, Uganda \\ ${ }^{2}$ Infectious Diseases Research Collaboration, Kampala, Uganda \\ Correspondence should be addressed to Violet Okaba Kayom; vokayom@gmail.com
}

Received 29 July 2015; Revised 15 November 2015; Accepted 18 November 2015

Academic Editor: Samuel Menahem

Copyright (c) 2015 Violet Okaba Kayom et al. This is an open access article distributed under the Creative Commons Attribution License, which permits unrestricted use, distribution, and reproduction in any medium, provided the original work is properly cited.

Background. Most information on newborn care practices in Uganda is from rural communities which may not be generalized to urban settings. Methods. A community based cross-sectional descriptive study was conducted in the capital city of Uganda from February to May 2012. Quantitative and qualitative data on the newborn care practices of eligible mothers were collected. Results. Over $99 \%$ of the mothers attended antenatal care at least once and the majority delivered in a health facility. Over $50 \%$ of the mothers applied various substances to the cord of their babies to quicken the healing. Although most of the mothers did not bathe their babies within the first 24 hours of birth, the majority had no knowledge of skin to skin care as a thermoprotective method. The practice of bathing babies in herbal medicine was common (65\%). Most of the mothers breastfed exclusively (93.2\%) but only $60.7 \%$ initiated breastfeeding within the first hour of life, while a significant number (29\%) used prelacteal feeds. Conclusion. The inadequate newborn care practices in this urban community point to the need to intensify the promotion of universal coverage of the newborn care practices irrespective of rural or urban communities and irrespective of health care seeking indicators.

\section{Introduction}

Neonatal morbidity and mortality have remained high in most developing countries and are affecting their attainment of Millennium Development Goal (MDG) 4. Neonatal sepsis is one of the major causes of these deaths, most of which are preventable $[1,2]$. Studies in developing countries have shown strong evidence that delivery and newborn care practices impact on neonatal sepsis [3-7]. In an effort to combat these alarming rates, World Health Organization (WHO) has put forth a set of evidence based neonatal health interventions called the essential newborn care (ENC) practices. They include clean delivery, cord care, skin and eye care, early and exclusive breastfeeding, thermal protection, and immunisation [8]. These interventions have been widely emphasized in policy and rolled out in developing countries.

Uganda is grappling with a high neonatal mortality rate of 27 per 1000 live births with neonatal sepsis being the major contributor [9]. Unhygienic delivery and poor newborn care practices coupled with poor accessibility to health facilities in rural areas have been cited as key risk factors $[9,10]$. Although urban communities in Uganda have better accessibility to health facilities and higher rates of antenatal care (ANC) attendance (98\%) and report more deliveries under skilled supervision [9], health facilities in urban settings continue to receive large numbers of neonates presenting with symptoms of neonatal sepsis. A survey done in Mulago National Referral Hospital in Uganda in 2012 showed that neonatal sepsis was the most common cause of admission to the neonatal ward (unpublished data). Most of these neonates were born in health facilities and discharged home, only to return to hospital with symptoms of neonatal sepsis. This finding suggests that there are unexplained risk factors not only in the health facilities but also in the communities to which these neonates are discharged.

Most studies on newborn care practices have been conducted in rural settings and these studies have reported disturbing rates of poor delivery and newborn care practices [11, 12]. There is a gap in information on newborn care practices in urban settings which are usually cross-cultural and better 
served with health care, yet reporting high rates of admission with neonatal sepsis. This study was therefore conducted in the capital city of Uganda and it sought to describe the newborn care practices among mothers in that community.

\section{Methodology}

2.1. Study Design. This was a community based cross-sectional descriptive study.

2.2. Study Site. The study was conducted in Kawempe division, one of the divisions of Kampala city which is the capital city of Uganda. Kawempe division has an area of 32.45 square kilometers and is administratively made up of 22 parishes and 119 zones. It is a densely populated area and according to the 2002 Population and Housing Census its population estimate was 268,659 of which $52 \%$ were female. The total number of households was 67,132. The division has several areas with uncontrolled developments and slum conditions. It is served by the National Referral Hospital, 2 government health centres, a private-not-for-profit hospital, and many privately owned clinics which provide curative services to the community [13].

2.3. Study Population. The study included all mothers of neonates residing within Kawempe division during the study period who met the selection criteria.

\subsection{Study Duration. The study was conducted from February} to May 2012 (4 months).

2.5. Inclusion Criteria. The inclusion criteria included all mothers of neonates living within Kawempe division during the study period who accepted to give informed consent to participate in the study.

2.6. Exclusion Criteria. The exclusion criteria included mothers of babies with gross congenital malformation.

2.7. Samples Size. The sample size was calculated using the modified Kish Leslie formula:

$$
N=\frac{\left(Z^{2 *} P(1-P) * \text { Deff }\right)}{D^{2}}
$$

where $Z$ is standard normal value corresponding to $95 \%$ confidence interval (1.96), $D$ is absolute error between the estimated and true value which equals $0.05(5 \%), P$ is estimated prevalence of poor newborn care practices which is $17 \%$, and Deff represents design effect taken to be 1.5 .

Hence, $N=325$ neonates.

2.8. Number of Clusters. The number of clusters, $C$, that was studied was calculated from the following formula [14]:

$$
C=\frac{P(1-P) D}{S^{2} b}
$$

where $P$ is prevalence, $D$ is design effect (1.5), $S$ is standard error given by confidence interval $/ Z$ alpha $(0.05 / 1.96=$ $0.0255)$, and $b$ is number of responses per cluster set at 10 for convenience.

The estimated number of clusters was 33. To allow for nonresponse a total of 34 clusters were studied. Thus the sample size calculated was 335 mothers with neonates.

2.9. Data Collection. Probability proportional to size was used to sample 34 villages out of 119 villages within Kawempe division in Kampala. The research team held meetings with chairpersons of the Local Council 1 and a member of the Village Health Team (VHT) of each sampled village to discuss the study. The VHT member guided the study team to the households in each village and households with neonates were identified. Informed consent was obtained from the eligible mothers of the neonates and a total of 10 households with neonates were consecutively enrolled from each village. A pretested questionnaire was used to record information on the characteristics of study participants and newborn care practices of the mothers. The gestational age and birth weight of the neonates were estimated by self-report. The newborn practices assessed included cord care (number of times the umbilical stump was cleaned in a day and what was applied on the umbilical stump), skin care (number of times the baby's skin was cleaned in a day, washing of hands prior to handling the baby, and use of herbal medicine to bath baby), early and exclusive breastfeeding, thermal protection, and immunisation.

The newborn care practices were further explored in a focus group discussion (FGD) which was conducted on completion of enrolment of the 338 study participants. Nine participants were included in the FGD. These were mothers of neonates who were identified from 2 zones within the study area by the VHTs with guidelines from the study team. Their ages ranged between 21 and 34 years. The FGD was moderated by the principal investigator assisted by a notetaker and the interviews were conducted in the local language of the majority of participants (Luganda) and English. The responses were translated into English by the note-taker and the principal investigator and the information was crosschecked to confirm the meanings.

2.10. Data Management and Analysis. All data was entered, cleaned, edited, coded, and double entered into ACCESS data base 2007. It was stored in both manual and electronic forms. Quantitative data was exported to STATA version 10 for analysis. Categorical variables were summarised into percentages and proportions.

Qualitative data from the FGD was stored in manual form. The information was compared and meaningful units were coded. These were broadly categorised into themes. Relevant quotes were extracted and represented.

2.11. Ethical Considerations. Written informed consent was obtained from the mothers of infants who were eligible.

Institutional approval was sought from Makerere University School of Medicine Research and Ethics Committee and the Uganda National Council for Science and Technology. 
TABLE 1: Characteristics of 338 mothers in Kampala District, Uganda, 2012.

\begin{tabular}{|c|c|c|}
\hline Variable & $\begin{array}{l}\text { Frequency } \\
\qquad(n)\end{array}$ & $\begin{array}{c}\text { Percentage } \\
\text { (\%) }\end{array}$ \\
\hline \multicolumn{3}{|l|}{ Attended antenatal care } \\
\hline Yes & 336 & 99.4 \\
\hline No & 2 & 0.6 \\
\hline \multicolumn{3}{|c|}{ Number of ANC attendances } \\
\hline$<4$ times & 172 & 51 \\
\hline$\geq 4$ times & 166 & 49 \\
\hline \multicolumn{3}{|l|}{ Marital status } \\
\hline Never married & 40 & 11.8 \\
\hline Divorced & 11 & 3.3 \\
\hline Married & 287 & 84.9 \\
\hline \multicolumn{3}{|l|}{ Parity } \\
\hline Prime & 88 & 26.0 \\
\hline $2-3$ & 164 & 48.5 \\
\hline$\geq 4$ & 86 & 25.5 \\
\hline \multicolumn{3}{|l|}{ Maternal education } \\
\hline None & 14 & 4.2 \\
\hline Primary & 124 & 36.7 \\
\hline Secondary & 160 & 47.3 \\
\hline Tertiary & 40 & 11.8 \\
\hline \multicolumn{3}{|c|}{ Received paternal financial support } \\
\hline Yes & 306 & 90.8 \\
\hline No & 32 & 9.5 \\
\hline \multicolumn{3}{|l|}{ Place of delivery } \\
\hline Hospital & 209 & 61.8 \\
\hline Health centre & 70 & 20.7 \\
\hline Clinic/maternity & 38 & 11.3 \\
\hline Home & 21 & 6.2 \\
\hline \multicolumn{3}{|c|}{ Duration of rupture of membranes } \\
\hline Less than 18 hours & 304 & 89.9 \\
\hline More than 18 hours & 34 & 10.1 \\
\hline
\end{tabular}

2.12. Study Profile. During the study period, a total of 358 mother-infant pairs were screened for the study. Fifteen did not consent to participate in the study, while 5 babies were found to be over 28 days. Therefore 338 mother-infant pairs were enrolled in the study.

\section{Results}

3.1. Description of the Study Participants. Twenty-six percent of the mothers were prime gravida. Their mean age was 25.4 years (sd 5.4).

Most of the mothers were married and received financial support from their husbands. Over $99 \%$ of the mothers attended ANC at least once and the majority delivered in a health facility (Table 1).

Most of the neonates were born at term and the mean gestational age was 39.7 weeks (sd 2.3) with mean birth weight of $3.4 \mathrm{~kg}$ (sd 0.7 ). Thirty-two (9.5\%) of the neonates had low birth weight. The neonates were equally distributed by gender with males comprising 156 (46.2\%) and the mean age at enrolment was 14.8 days (sd 8.5).

3.2. Newborn Care Practices of Mother-Infant Dyads. The newborn care practices assessed in this study included cord care, thermal protection, skin care, early and exclusive breastfeeding, and immunisation.

3.2.1. Cord Care. More mothers reported that they had received teachings on cord care $(69.5 \%)$ although few $(45 \%)$ had received the teachings from health personnel. Some mothers reportedly received teachings from their attendants, neighbours, and fellow mothers. Majority of the mothers cleaned the cord at least twice a day $(89.6 \%)$ and washed their hands prior to cleaning the cord (84.9\%). About 58\% of the mothers applied various substances on the cord of their babies (Table 2). These substances included salty water, powder, Vaseline, spirit, normal saline, ripe banana (gonja), sap, soot, ash, saliva, and herbs.

Mothers who delivered in private clinics were 1.2 times more likely to apply substances to the cord compared to those who delivered in hospital. Incidentally there was no significant difference in the practice of application of substances to cord among mothers who delivered at home and those who delivered in hospital. The level of education, parity, and the number of ANC attendances did not result in any significant difference in the practice of application of substances to the cord (Table 3 ).

From the qualitative data majority of the mothers reported that it was important to apply substances to the cord to quicken the healing. The commonly used substance was warm salty water.

FGD member said, "I clean the baby's cord with clean cotton wool dipped in warm salty water which I keep in a bottle."

FGD member said, "Spirit helps the cord dry faster."

3.2.2. Thermal Protection. About sixty percent of the mothers did not bathe their babies within the first 24 hours of birth. However, most of the mothers had no knowledge of skin to skin care or kangaroo mother care (KMC) as a way of keeping babies warm (77\%). Only $28 \%$ of the mothers with low birth weight or preterm babies practiced KMC (Table 2).

Mothers who delivered in health centres and private clinics were twice more likely to bathe their babies within 24 hours of birth compared to those who delivered in hospital. Incidentally those who delivered at home were 1.5 times less likely to bathe their babies within 24 hours of birth compared to those who delivered in health centres or private clinics (Table 3).

In the qualitative data, all the mothers acknowledged the importance of keeping babies warm in the first 24 hours. However, some of the reasons given for thermal protection were not the scientifically valid reasons, while other mothers 
TABLE 2: The newborn care practices of 338 mother-infant dyads in Kampala District, Uganda, 2012.

\begin{tabular}{|c|c|c|}
\hline Variable & $\begin{array}{l}\text { Frequency } \\
\qquad(n)\end{array}$ & $\begin{array}{c}\text { Percentage } \\
(\%)\end{array}$ \\
\hline \multicolumn{3}{|c|}{ Applied substance on the cord } \\
\hline No & 141 & 41.7 \\
\hline Yes & 197 & 58.3 \\
\hline \multicolumn{3}{|c|}{$\begin{array}{l}\text { Number of times the cord is cleaned per } \\
\text { day }\end{array}$} \\
\hline None & 22 & 6.5 \\
\hline Once & 13 & 3.8 \\
\hline$\geq 2$ times & 303 & 89.6 \\
\hline \multicolumn{3}{|c|}{$\begin{array}{l}\text { Washing of hands before cleaning the } \\
\text { cord }\end{array}$} \\
\hline No & 51 & 15.1 \\
\hline Yes & 287 & 84.9 \\
\hline \multicolumn{3}{|l|}{ Taught cord care } \\
\hline No & 103 & 30.5 \\
\hline Yes & 235 & 69.5 \\
\hline \multicolumn{3}{|c|}{ Bathed baby within 24 hours } \\
\hline No & 201 & 59.5 \\
\hline Yes & 137 & 40.5 \\
\hline \multicolumn{3}{|c|}{ Knew skin to skin/kangaroo mother care } \\
\hline No & 261 & 77.2 \\
\hline Yes & 77 & 22.8 \\
\hline \multicolumn{3}{|c|}{$\begin{array}{l}\text { Washing of hands prior to handling the } \\
\text { baby }\end{array}$} \\
\hline No & 56 & 16.6 \\
\hline Yes & 280 & 83.4 \\
\hline \multicolumn{3}{|c|}{$\begin{array}{l}\text { Number of times the baby is cleaned in a } \\
\text { day }\end{array}$} \\
\hline Less than twice & 33 & 9.8 \\
\hline Two or more times & 305 & 90.2 \\
\hline \multicolumn{3}{|c|}{ Bathe baby with herbal medicine } \\
\hline No & 113 & 34.4 \\
\hline Yes & 205 & 60.7 \\
\hline \multicolumn{3}{|l|}{ Exclusive breastfeeding } \\
\hline No & 23 & 6.8 \\
\hline Yes & 315 & 93.2 \\
\hline \multicolumn{3}{|c|}{ Initiation of breastfeeding } \\
\hline Less than 1 hour & 205 & 60.7 \\
\hline More than 1 hour & 133 & 39.3 \\
\hline \multicolumn{3}{|l|}{ Use of prelacteal feeds } \\
\hline No & 238 & 70.4 \\
\hline Yes & 100 & 29.6 \\
\hline \multicolumn{3}{|l|}{ Immunisation } \\
\hline No & 79 & 23.4 \\
\hline Yes & 259 & 76.6 \\
\hline
\end{tabular}

did not know why they delayed bathing their babies. Examples of misconceptions and lack of knowledge about reason for practices are reflected in the quotes below.
FGD member said, "You keep the baby warm because you do not want cold air to enter the baby's lungs since it can cause pneumonia."

FGD member said, "I bathe my baby 2 days after birth because the baby collapses in water if you bathe early."

FGD member said, "I do not know why I have to wait for 2 days to bathe my baby."

3.2.3. Skin Care. Majority of mothers washed their hands prior to handling their babies $(83.4 \%)$ and cleaned their babies at least twice in a day (90.2\%). The practice of bathing babies in herbal medicine was common in majority of the respondents, that is, $205(60.7 \%)$. In the qualitative study, there were conflicting views with regard to bathing babies in herbal medication which was called "ekyogero." This was said to be a mixture of various herbs including leaves, roots, and tree barks. The herbal medications were used to prevent and treat skin rashes (locally called "nnoga"), treat abdominal colic, and give the baby good luck. Examples of practices and beliefs about the use of herbal medicines to bathe the baby as reported in the FGD are shown by the quotes below.

FGD member said, "Before you bathe the baby in ekyogero you drop some of the fluid in the baby's mouth. This prevents the baby from suffering from stomache [sic] pain."

FGD member said, "Bathing the baby in ekyogero makes the baby get good luck. Anyway other children are not bathed in it but they get their blessings from God."

Other members did not agree with the practice of bathing babies in herbal medicine mainly due to cultural differences.

FGD member said, "For us the Bakiga we do not bathe our babies in ekyogero, we just bathe the baby with soap and apply powder and vaseline. But look we are among the luckiest people...."

3.2.4. Breastfeeding. Most of the mothers breastfed their babies exclusively (93.2\%), while only $60.7 \%$ initiated breastfeeding within the first hour of life (Table 2). Over twentynine percent $(29.6 \%)$ of the mothers used prelacteal feeds which included glucose solution, cow's milk, water, and tea.

Mothers who delivered in hospitals or health centres were 1.2 times more likely to initiate breastfeeding within the first hour of life compared to those who delivered at home or in private clinics. Level of education, parity, and number of ANC attendances were not found to significantly affect time to initiation of breastfeeding (Table 3).

In the FGD there were varying views about the time to initiate breastfeeding following delivery. Some mothers said it was important to put the baby to breastfeed immediately after birth, while others thought there were no strict timelines. It was also reported that health workers take the babies away for long and this delays initiation of breastfeeding. Most mothers 
TABLE 3: Levels of selected newborn care practices in selected demographic, antenatal, and delivery characteristics of mother-infant dyads in Kampala district, Uganda, 2012.

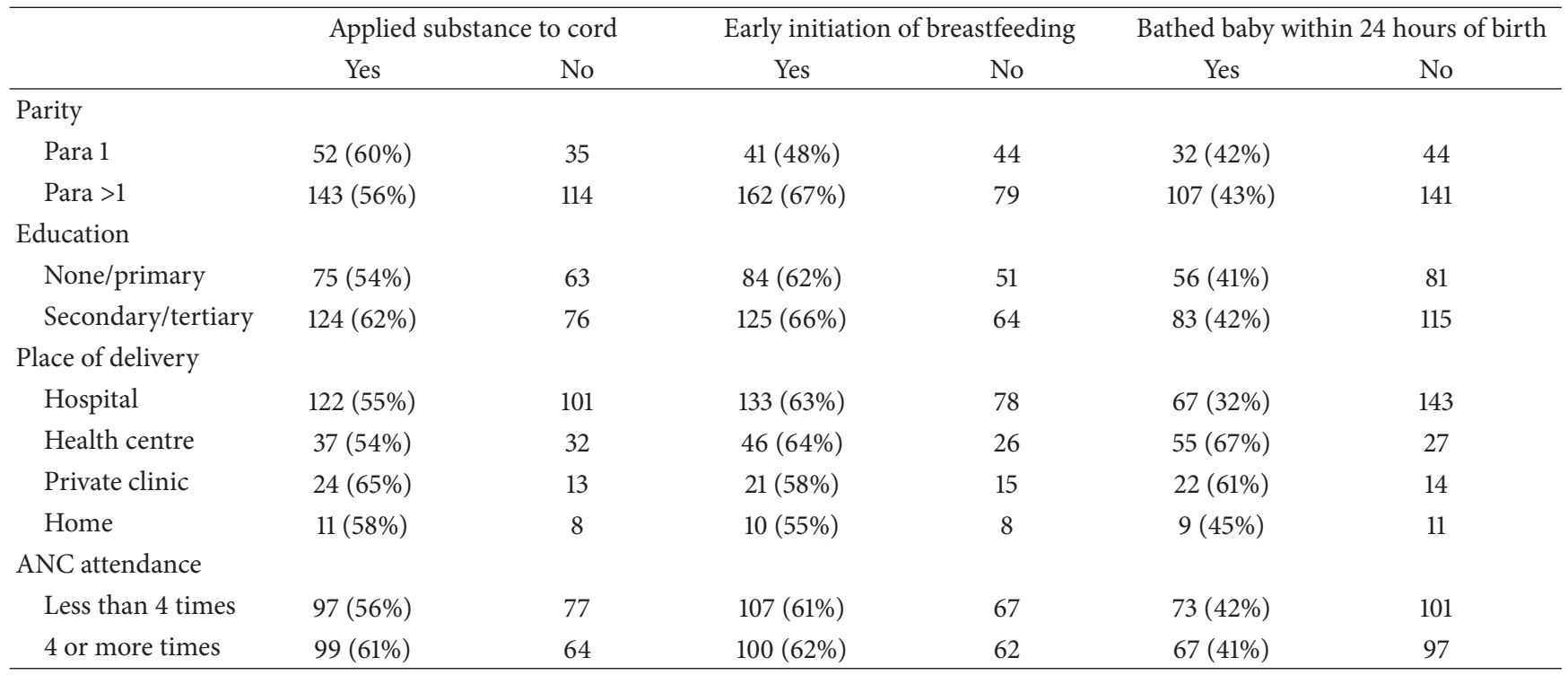

thought it was okay to give prelacteal feeds although a few strongly objected. Examples of breastfeeding practices and misconceptions are reflected in the quotes below.

\section{FGD member said, "You should put the baby immediately on the breast even if there is no milk."}

FGD member said, "The health workers sometime take the baby away from you, so it is okay to wait."

FGD member said, "You give the baby warm water with glucose because you may have no breast milk...." (Most mothers agreed to this.)

3.2.5. Immunisation. Seventy-six percent of the babies had received their Bacille Calmette-Guérin (BCG) and Oral Polio Vaccine (OPV) at the time of contact with the study team.

\section{Discussion}

This study was conducted to determine the newborn care practices among mother-infant dyads in an urban community in Uganda. Although some components of the evidence based newborn care practices in this study were appropriate, the overall practices were deemed inadequate.

4.1. Cord Care. In our study, majority of the mothers cleaned the cord frequently and washed their hands prior to cleaning the cord. However, the practice of applying substances on the cord was highly prevalent. It was quite surprising to find that the practice of application of substances to the cord was equally prevalent among mothers who delivered in health facility and those who delivered at home. This suggests that the teachings on appropriate cord care at the health facilities are not adequate or the uptake is poor possibly due to cultural beliefs. From the qualitative data it was clear that most mothers believe that various substances should be applied to the cord to hasten healing of the stump. This finding is similar to that reported in rural Eastern Uganda in which about half of the mothers applied various substances to the cord [11]. However, a study in urban Nepal reported much better cord care with $86 \%$ of the mothers who had delivered in health facilities not applying any substance to the cord and rates of $63 \%$ of those who had delivered at home [15]. The difference in the findings may be due to cultural variations between the urban community in our study and that in Nepal. WHO currently recommends that the cord be cleaned with daily chlorhexidine for babies born at home in high mortality settings. For babies born in health facilities or at home in low mortality settings it may be considered to replace application of harmful traditional substances [16]. Our study indicates that there are a knowledge gap and misconceptions on care of the cord in this urban community and the evidence based recommendations have not been taken up. It is very surprising that although over $90 \%$ of the mothers in our study attended ANC and the majority delivered in a health facility, only about $45 \%$ received teachings on appropriate cord care from health personnel. There is no doubt that the mothers are not knowledgeable on the various aspects of appropriate cord care since they do not have a clear expert source of information. The health facilities should therefore step up the health education on appropriate cord care during ANC and in the period after delivery. In addition, WHO recommendation for chlorhexidine cleansing should be extended fully to all babies in settings with high mortality and high infection regardless of place of birth.

4.2. Thermal Protection. Most of the mothers in this community did not have any knowledge of skin to skin care or $\mathrm{KMC}$, and even for mothers with low birth weight or preterm babies most did not practice KMC. KMC has been shown to reduce neonatal mortality among low birth weight and preterm babies and reduce severe morbidity [3]. This practice 
should be widely scaled up especially in high mortality, low resource settings.

Some components of good thermal protection were practiced by majority of the participants. These included delayed bathing for more than 24 hours and covering babies in warm clothes immediately after birth. Although mothers who delivered in hospitals were less likely to bathe their babies within 24 hours of birth compared to those who delivered in health centres or private clinics, mothers who delivered at home were equally less likely to bathe their babies within 24 hours of birth. This finding shows that place of delivery does not necessarily result in good thermal practice and thus the promising finding of delayed bathing in majority of the mothers may simply be due to cultural practice and may not necessarily be a result of health facility promotion of the evidence based practices. This therefore questions the adequacy of teachings on thermal protection. WHO recommends that babies be bathed after 24 hours of birth and not earlier to avoid hypothermia [8]. A study in rural eastern Uganda reported that only $42 \%$ of the neonates received adequate thermal protection. In that community early bathing was practiced because of the cultural belief that babies are born dirty [11]. Thermal protection in various communities in Africa seems to vary mainly due to cultural differences. A large crosssectional study in Tanzania reported that two-thirds of the mothers bathed their babies within 6 hours of delivery and $10 \%$ reported that their babies were dipped in cold water immediately after birth [17]. The health facility delivery rate in that study was $40 \%$ suggesting poor health seeking behaviour which could also explain the poor thermal protection practice. The subjects in our study had diverse cultural origins and this possibly could have had a dilutional effect on the practice, thus exposing the participants in our study to better thermal protection practice.

Although most of the mothers acknowledged the importance of keeping the baby warm, the qualitative data shows that the majority did not know the scientifically valid reasons why these practices are encouraged. Some mothers perform practices which are important in thermal protection but they do not know that these practices are actually meant to keep the baby warm. An example is delayed bathing of the baby after birth. If mothers do not know why they are being told to do certain things, they may not own the practices and this may affect compliance with the evidence based practices.

4.3. Skin Care. Majority of the mothers in our study cleaned their babies two or more times a day. However, the practice of bathing babies in herbal medications (ekyogero) was common. The herbal medication is a concoction of various roots, leaves, and stems of plants and is used to bathe the baby, applied to the cord, and given to the baby to drink. The use of this herbal medication has been reported in other communities in Uganda. The health impact of such a practice is not well investigated, yet it is strongly embedded in the culture of this urban community. Further studies on the safety and health effect of use of the herbs on the newborn are therefore warranted.

Hand washing was practiced by a significant number of mothers in our study. Maternal and birth attendant hand washing has been shown to reduce infection in neonates [5] and so its promotion should be intensified in the community.

4.4. Early Initiation and Exclusive Breastfeeding. Majority of the mothers in our study practiced exclusive breastfeeding suggesting that some components of the ENC practices have been embraced well in this community. This study reports better exclusive breastfeeding rates compared to that reported in Southern Tanzania in which less than half breastfed exclusively in the 3 days after delivery [17]. The difference in these results may be due to the better health facility delivery in our study.

Our study showed that just over half of the mothers initiated breastfeeding within the first hour of life and about a third gave prelacteal feeds. The finding that mothers who delivered in hospitals and health centres were more likely to initiate breastfeeding within the first hour of life compared to those who delivered in private clinics or at home suggests that there is inadequate promotion of the ENC practices in private facilities and the community. A study in Ghana showed that early initiation of breastfeeding could avert $22 \%$ of neonatal deaths [4]. WHO discourages the use of prelacteal feeds unless medically indicated because of its association with insufficient milk production, infection transmission, and lactation failure [18]. Our findings show that despite the good exclusive breastfeeding rates some key components of breastfeeding are still lacking and there is a gap in the private health facilities. The promotion of breastfeeding should therefore focus not only on exclusive breastfeeding but also on early initiation of breastfeeding and discourage the use of prelacteal feeds in the community and public and private health facilities.

4.5. Immunisation. In our study, $76 \%$ of the neonates had received BCG and OPV at the time of contact with the research team. According to the Uganda National Expanded Program for Immunisation (UNEPI) all infants should receive BCG and OPV at birth or within 2 weeks of delivery. According to the Uganda Demographic Health Survey (UDHS) 2011, 96\% of neonates in urban settings received BCG as compared to $93 \%$ in rural settings [9]. The immunisation rate in our study is therefore lower than that reported in the UDHS 2011. This indicates that a significant number of babies born in health facilities are being discharged without receiving their $B C G$ and OPV vaccinations. This finding suggests that the health facilities do not have constant supply of vaccines or there is inadequate promotion of the vaccination. The Ministry of Health of Uganda $(\mathrm{MoH})$ and health partners should therefore ensure that every public health facility offers immunisation services daily. Community outreach should be supported and promoted to mop up the remnants of infants who missed the vaccinations in health facilities. The private health facilities should also be supported to provide immunisation services.

4.6. Overall Newborn Care Practices. This study was conducted in an urban community which is well served with health facilities. The ANC attendance of the mothers was very high and the rate of health facility delivery and skilled birth 
attendance was almost twice the national rate and much higher than that reported in the studies in rural settings $[9,11$, 12]. It is therefore quite surprising that most of the newborn care practices were not any better than those reported in the rural settings. It is clear that the good health seeking indicators of this community have not really translated into gains in the newborn care practices. The $\mathrm{MoH}$ and partners should consider reviewing the strategies currently being used to disseminate the newborn care practices in health facilities and communities. This should include review of the content, mode, and intensity of health education on newborn care practices by health workers during ANC, after delivery, and in postnatal follow-up and involvement of VHTs in the newborn care health education. In addition, the health effects of the cultural practice of bathing babies in the herbal concoction and dropping the fluid in the babies' mouths in this community should be investigated.

4.7. Study Limitations. The gestational age and birth weight of the neonates were estimated by self-report from the mothers and this may not be accurate.

\section{Conclusion}

Although some components of the evidence based newborn care practices in this urban community were appropriate, the overall practices were deemed inadequate. The strategies which are currently being used to disseminate the newborn care practices are not meeting the stated objectives. There is therefore pressing need to review and intensify the promotion of universal coverage of the newborn care practices irrespective of rural or urban communities, place of delivery, and the health care seeking indicators being reported.

$\begin{array}{ll}\text { Abbreviations } \\ \text { ANC: } & \text { Antenatal care } \\ \text { BCG: } & \text { Bacille Calmette-Guérin } \\ \text { ENC: } & \text { Essential newborn care practices } \\ \text { FGD: } & \text { Focus group discussion } \\ \text { KMC: } & \text { Kangaroo mother care } \\ \text { MDG: } & \text { Millennium Development Goal } \\ \text { MoH: } & \text { Ministry of Health of Uganda } \\ \text { OPV: } & \text { Oral Polio Vaccine } \\ \text { UDHS: Uganda Demographic Health Survey } \\ \text { UNEPI: Uganda National Expanded Program for } \\ \text { VHT: } \quad \text { Village Health Team } \\ \text { WHO: }\end{array}$

\section{Conflict of Interests}

All the authors declare that there is no conflict of interests regarding the publication of this paper.

\section{Authors' Contribution}

Violet Okaba Kayom conceived the study, participated in its design, coordinated the study, drafted the initial paper, and approved the final paper as submitted. Abel Kakuru carried out the initial statistical analysis, reviewed and revised the paper, and approved the final paper as submitted. Sarah Kiguli participated in the design of the study, reviewed and revised the paper, and approved the final paper as submitted.

\section{Acknowledgments}

The authors would like to thank all the mothers and babies who participated in this study. They acknowledge the contribution of the research assistants, the Village Health Teams, and Local Council chairpersons of the zones they visited during the data collection.

\section{References}

[1] R. E. Black, S. Cousens, H. L. Johnson et al., "Global, regional, and national causes of child mortality in 2008: a systematic analysis," The Lancet, vol. 375, no. 9730, pp. 1969-1987, 2010.

[2] J. E. Lawn, K. Wilczynska-Ketende, and S. N. Cousens, "Estimating the causes of 4 million neonatal deaths in the year 2000," International Journal of Epidemiology, vol. 35, no. 3, pp. 706-718, 2006.

[3] J. E. Lawn, J. Mwansa-Kambafwile, B. L. Horta, F. C. Barros, and S. Cousens, "Kangaroo mother care' to prevent neonatal deaths due to preterm birth complications," International Journal of Epidemiology, vol. 39, supplement 1, pp. i144-i154, 2010.

[4] K. M. Edmond, C. Zandoh, M. A. Quigley, S. Amenga-Etego, S. Owusu-Agyei, and B. R. Kirkwood, "Delayed breastfeeding initiation increases risk of neonatal mortality," Pediatrics, vol. 117, no. 3, pp. e380-e386, 2006.

[5] V. Rhee, L. C. Mullany, S. K. Khatry et al., "Maternal and birth attendant hand washing and neonatal mortality in southern Nepal," Archives of Pediatrics and Adolescent Medicine, vol. 162, no. 7, pp. 603-608, 2008.

[6] R. Ghosh and A. K. Sharma, "Determinants of tetanus and sepsis among the last neonatal deaths at household level in a periurban area of India," Postgraduate Medical Journal, vol. 87, no. 1026, pp. 257-263, 2011.

[7] K. M. Edmond, B. R. Kirkwood, S. Amenga-Etego, S. OwusuAgyei, and L. S. Hurt, "Effect of early infant feeding practices on infection-specific neonatal mortality: an investigation of the causal links with observational data from rural Ghana," American Journal of Clinical Nutrition, vol. 86, no. 4, pp. 1126-1131, 2007.

[8] I. Narayanan, M. Rose, D. Cordero, S. Faillace, and T. Sanghvi, The Components of Essential Newborn Care, The Basics Support for Institutionalizing Child Survival Project (BASICS II) for the United States Agency for International Development, Arlington, Va, USA, 2004.

[9] Uganda Bureau of Statistics (UBOS) and ICF International, Uganda Demographic and Health Survey 2011, UBOS and Calverton, Maryland: ICF International, Kampala, Uganda, 2012.

[10] Ministry of Health. Uganda Government, Situation Analysis of Newborn Health in Uganda, Ministry of Health, Uganda Government, Kampala, Uganda, 2008.

[11] P. Waiswa, S. Peterson, G. Tomson, and G. W. Pariyo, "Poor newborn care practices-a population based survey in eastern Uganda," BMC Pregnancy and Childbirth, vol. 10, article 9, 2010. 
[12] R. N. Byaruhanga, J. Nsungwa-Sabiiti, J. Kiguli, A. Balyeku, X. Nsabagasani, and S. Peterson, "Hurdles and opportunities for newborn care in rural Uganda," Midwifery, vol. 27, no. 6, pp. 775-780, 2011.

[13] S. Lwasa and J. B. Nyakana, "Development planning and implementation processes for employment, health services and housing provision in Kawempe division, Kampala district," NURRU Report, 2005.

[14] S. Bennett, T. Woods, W. M. Liyanage, and D. L. Smith, "A simplified general method for cluster-sample surveys of health in developing countries," World Health Statistics Quarterly, vol. 44, no. 3, pp. 98-106, 1991.

[15] C. T. Sreeramareddy, H. S. Joshi, B. V. Sreekumaran, S. Giri, and N. Chuni, "Home delivery and newborn care practices among urban women in western Nepal: a questionnaire survey," BMC Pregnancy and Childbirth, vol. 6, article 27, 2006.

[16] World Health Organization, WHO Recommendations on Postnatal Care of the Mother and Newborn, World Health Organization, Geneva, Switzerland, 2013, http://apps.who.int/iris/ bitstream/10665/97603/1/9789241506649_eng.pdf.

[17] S. Penfold, Z. Hill, M. Mrisho et al., "A large cross-sectional community-based study of newborn care practices in Southern Tanzania," PLoS ONE, vol. 5, no. 12, Article ID e15593, 2010.

[18] WHO/UNICEF, Innocent Declaration on the Protection, Promotion and Support of Breastfeeding in the 1990s, A Global Initiative, UNICEF, New York, NY, USA, 1990. 


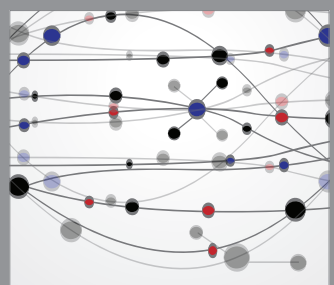

The Scientific World Journal
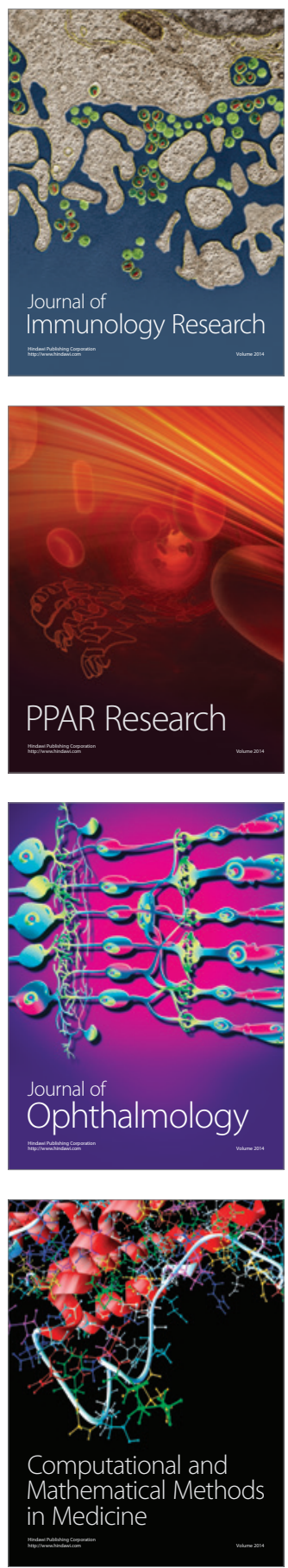

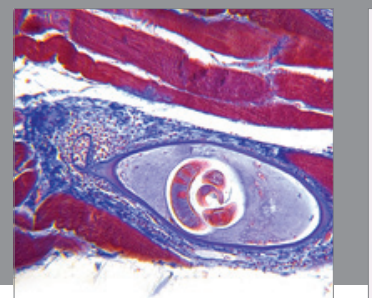

Gastroenterology

Research and Practice
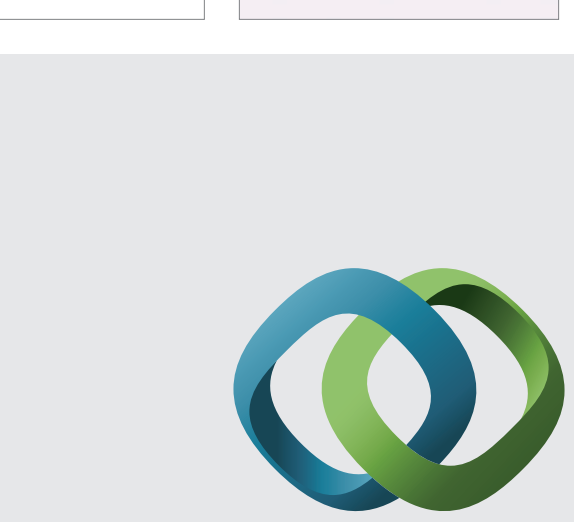

\section{Hindawi}

Submit your manuscripts at

http://www.hindawi.com
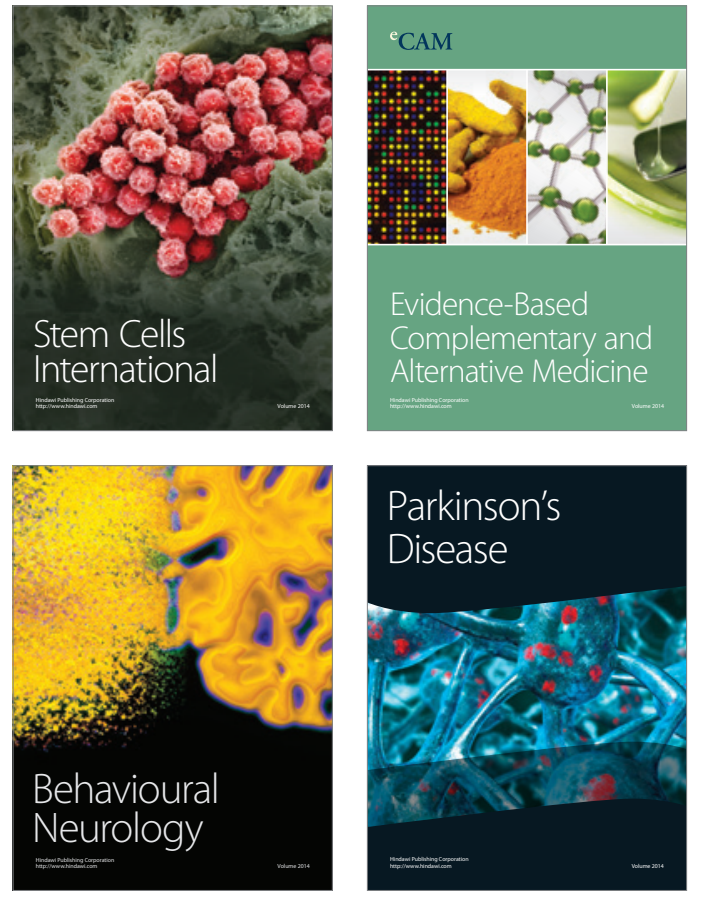
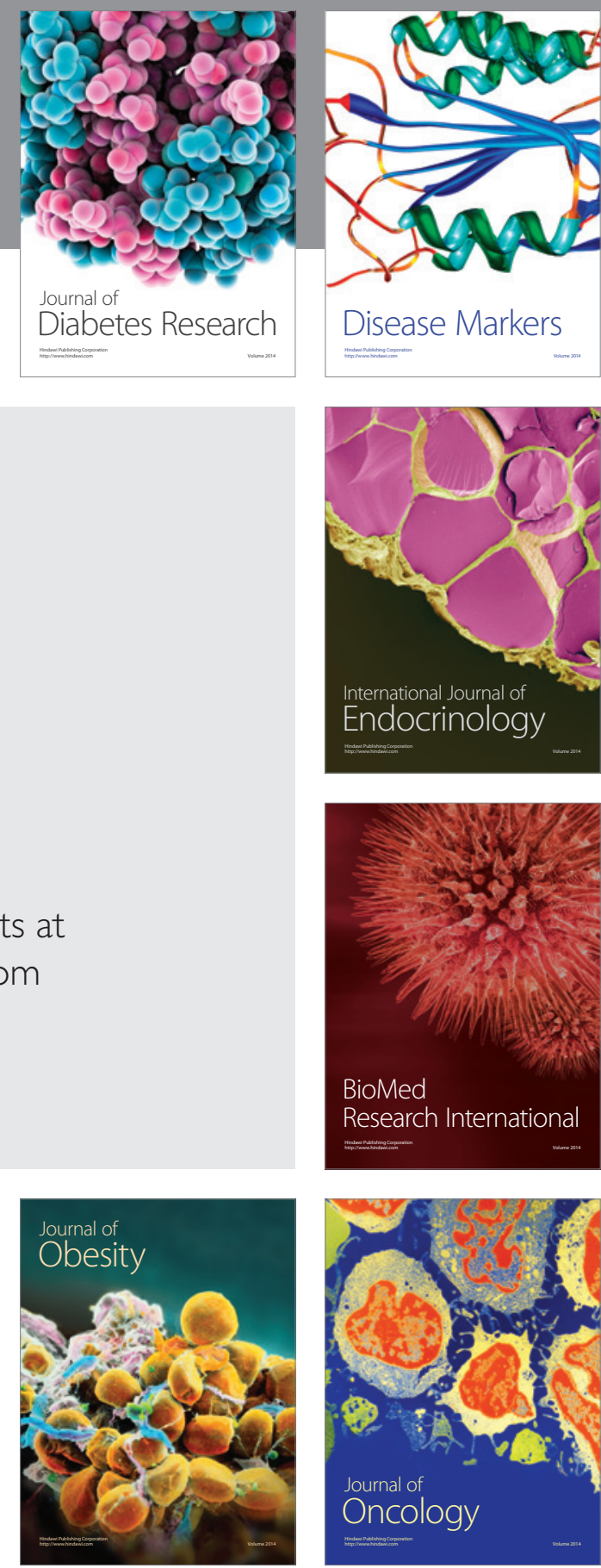

Disease Markers
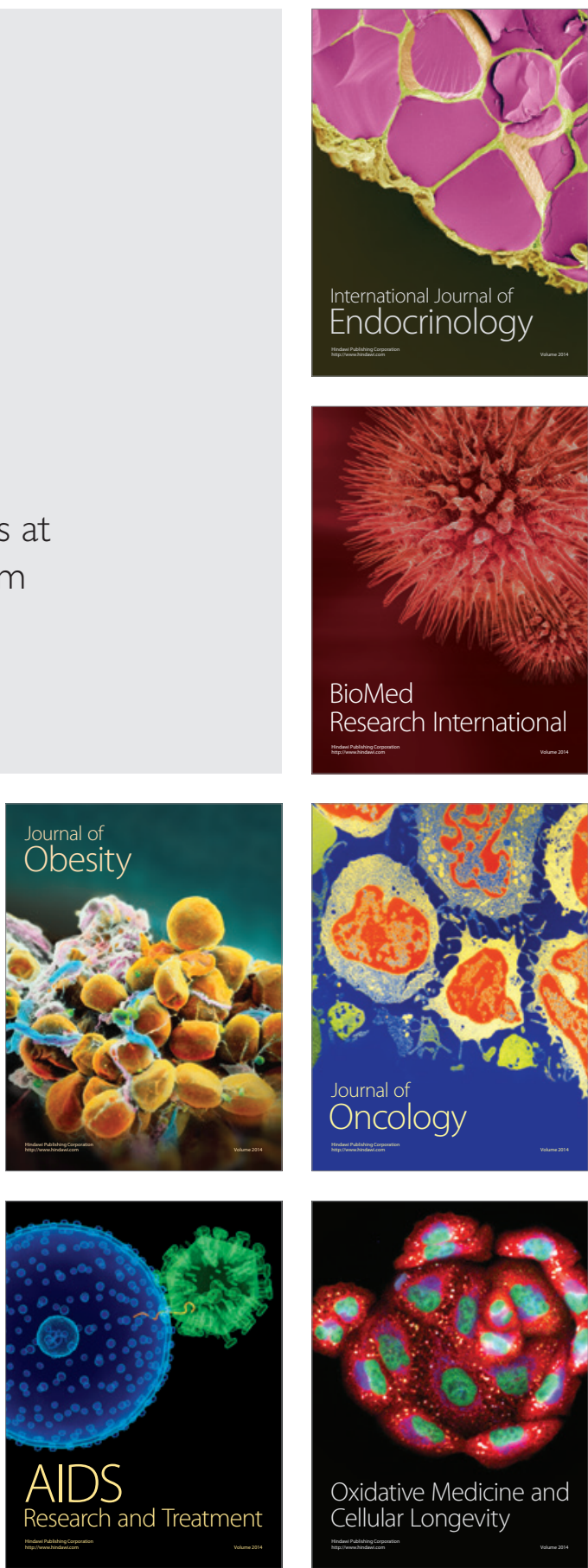